Research Paper

\title{
An Inhibitor of Casein Kinase $1 \varepsilon / \delta$ (PF670462) Prevents the Deterioration of Dextran Sodium Sulfate-induced Ulcerative Colitis Caused by UVB Eye Irradiation
}

\author{
Keiichi Hiramoto $^{1 凶}$, Yurika Yamate ${ }^{1}$, Emiko Kasahara ${ }^{2}$, Eisuke F Sato ${ }^{1}$ \\ 1. Department of Pharmaceutical Sciences, Suzuka University of Medical Science, Suzuka, Mie, Japan \\ 2. Department of Pharmaceutical Sciences, Osaka University Graduate School, Suita, Osaka, Japan \\ $\triangle$ Corresponding author: Keiichi Hiramoto, Ph.D; Department of Pharmaceutical Sciences, Suzuka University of Medical Science, 3500-3 Minamitamagakicho, \\ Suzuka, Mie 513-8670, Japan; Tel: +81-59-340-0575; Fax: +81-59-368-127; E-mail: hiramoto@suzuka-u.ac.jp \\ (C) Ivyspring International Publisher. This is an open access article distributed under the terms of the Creative Commons Attribution (CC BY-NC) license \\ (https://creativecommons.org/licenses/by-nc/4.0/). See http://ivyspring.com/terms for full terms and conditions.
}

Received: 2017.12.27; Accepted: 2018.04.01; Published: 2018.06.02

\begin{abstract}
Although we previously reported the exacerbation of dextran sodium sulfate (DSS)-induced ulcerative colitis by ultraviolet (UV) B eye irradiation, we do not yet understand the mechanism behind this phenomenon. In this study, we examined the relationship between the deterioration of DSS-induced ulcerative colitis and clock genes. We induced a mouse model of ulcerative colitis by administering DSS for 5 days, and administered UVB eye irradiation on each day of the DSS treatment period. The DSS-induced ulcerative colitis was deteriorated by the UVB eye irradiation. The levels of Clock, brain and muscle arnt-like protein 1 (Bmal1), reverse orientation c-erb A gene (Rev-Erb)a, RAR-related orphan receptor gamma (RORYt), and interleukin (IL)-17 in the colon were increased by UVB eye irradiation in the DSS-treated mice (UVB/DSS-treated mice). Conversely, the nuclear factor, interleukin 3 regulated (NFIL-3) levels in the colon were lower after UVB eye irradiation. The Casein Kinase $1 \varepsilon / \delta$ inhibitor (PF670462) administration, which is a Clock/Bmall inhibitor (PER2 activator), inhibited the deterioration caused by UVB eye irradiation. These results suggest that the UVB eye irradiation-mediated exacerbation of DSS-induced ulcerative colitis depends on IL-17 produced in response to alterations in clock genes.
\end{abstract}

Key words: Ultraviolet B; DSS-induced ulcerative colitiss; Clock genes; IL-17

\section{Introduction}

Ultraviolet (UV) rays have many effects on a living body, including activation of the immune system. For example, UV irradiation can induce photo-immunosuppression $(1,2)$ or skin cancer (3). The inhibitory action of UV irradiation on the immune system in the skin is thought to be mediated by regulatory $\mathrm{T}$ cells, which are induced by UV (4), decreases in the numbers and function of Langerhans cells (LCs) $(5,6)$, and the production of inhibitory cytokines and prostaglandin E2 by keratinocytes (7). Furthermore, there are many reports on the relationship between UV exposure and allergic diseases. In the atopic dermatitis (AD), the increase in active oxygen upon exposure to UV irradiation exacerbates atopic symptoms (8). On the other hand, radiation with narrow-band UVB $(311 \mathrm{~nm} \pm 2 \mathrm{~nm})$ is used to control AD by reducing the numbers of LCs, which are responsible for inducing the allergic reactions (9). Therefore, narrow-band UVB is used to treat $\mathrm{AD}$ and psoriasis.

We previously reported a deterioration of the autoimmune disease ulcerative colitis caused by UVB eye irradiation in the dextran sodium sulfate (DSS)induced mouse model (10). UVB eye irradiation stimulates the secretion of the hormone proopiomelanocortin (POMC), the precursor to adrenocorticotropic hormone $(\mathrm{ACTH})$, by the brain; the hormone increases inflammatory cytokine and thymic 
stromal lymphopoietin (TSLP) expression in the colon, resulting in the exacerbation of ulcerative colitis. However, this mechanism does not fully explain the deterioration of DSS-induced ulcerative colitis upon exposure to UVB eye irradiation.

The clock genes are involved in many inflammatory diseases. For example, increases in period circadian regulator 2 (PER2) and decreases in Clock/brain and muscle arnt-like protein 1 (Bmal1) can inhibit an immunoglobulin E (IgE)-dependent allergy (11). Clock genes are also related to pollinosis or asthma $(12,13)$. Thus, the circadian rhythm clock genes are involved in the deterioration of allergy symptoms. Therefore, the inflammation by an allergy may be controllable by changing expression of Bmal1, Clock, or PER2. Casein Kinase $1 \varepsilon / \delta(\mathrm{CK} 1 \varepsilon / \delta)$ is an essential component of the biological clock, phosphorylateing PER proteins. Consequently, by using $\mathrm{CK} 1 \varepsilon / \delta$ inhibitor, PER2 is activated and, as a result, Clock/Bmal1 is inhibited (14). From this, in this experiment, in order to observe a function of clock genes, we used $\mathrm{CK} 1 \varepsilon / \delta$ inhibitor. In addition, the circadian rhythm clock genes have a close relationship with the UV in sunlight (15). However, the relationship between the clock genes and the effects of UVB eye irradiation on ulcerative colitis is not clear.

In this study, we examined the role of the clock genes in the deterioration of DSS-induced ulcerative colitis caused by UVB eye irradiation.

\section{Materials and methods}

\section{Animals}

The male C57BL/6J mice (8-week-old) were purchased from SLC (Hamamatsu, Shizuoka, Japan). The mice were housed under specific-pathogen-free conditions and individually in cages in an airconditioned room maintained at $23 \pm 1^{\circ} \mathrm{C}$ with a 12-h light and 12-h dark cycle (lights on at 8:00 AM). Mice were divided to three groups (control group, DSS-treated group, and UVB/DSS treated group). Each group consisted of at least six mice. All experimental procedures described in his study were conducted according to the recommendations of the guide for the care and use of laboratory animals of Suzuka University of Medical Science (approval number: 34). All surgeries were performed under pentobarbital anesthesia, and all efforts were made to minimize suffering.

\section{UV irradiation}

The UVB source comprised a bank of sunlamps (FL20SE sunlamp, Toshiba, Tokyo, Japan) that there energy in the UVB range (280-320 nm) with an emission peak at $305 \mathrm{~nm}$. During the experimental period, the eye of mice was locally exposed UVB at a dose of $1.0 \mathrm{~kJ} / \mathrm{m}^{2} /$ day (irradiation time: $60 \mathrm{~s} /$ day) with light nembutal anesthesia. We irradiated to mice at zeitgeber time (ZT)2 (10). The rest of the body surface without eye was protected from irradiation with aluminum foil. The procedure was described in detail in previous our studies $(16,17)$. In the control group, the eye of mice was irradiated with visible light (FL20SD light source; wavelength: 400-700 nm, Toshiba, Tokyo, Japan).

\section{DSS-induced colitis}

For inducing ulcerative colitis, we administered $5.0 \%$ (w/v) DSS (molecular weight: 36,000-50,000 Da; MP Biomedicals, Solon, OH, USA) drinking water of mice in the DSS-treated group for five consecutive days. The development of colitis was monitored in each mouse by observing the condition of its feces. The severity of colitis was determined by the feces condition and post mortem colon length. The fecal condition score was determined using two parameters: stool consistency $(0=$ negative; $1=$ soft; $2=$ very soft, but formed; $3=$ liquid $)$ and fecal bleeding $(0$ $=$ negative; 1 = faintly blue; $2=$ moderately blue; 3 = dark blue; $4=$ blood visible using the guaiac paper test). The sum of two parameters was considered to be the individual disease activity score (18).

\section{4-[1-Cyclohexyl-4-(4-fluorophenyl)-1 H-imidaz ol-5-yl]-2-pyrimidinamine dihydrochloride (PF670462) treatment}

We used 4-[1-Cyclohexyl-4-(4-fluorophenyl)1H-imidazol-5-yl]-2-pyrimidinamine dihydrochloride (PF670462, Abcam, Tokyo, Japan) to inhibit Clock/ Bmal1 activity in mice. PF670462 is a potent, selective Casein Kinase $1 \varepsilon(\mathrm{CK} 1 \varepsilon)$ and CK1 $1 \delta$ inhibitor and PER2 activator. We subcutaneously administered PF670462 $(50 \mathrm{mg} / \mathrm{kg} /$ day) suspended in saline on each day of the experiment (14).

\section{Western blotting analysis}

We obtained the colon samples of mice at five days (at morning), and these were homogenized in Lysis Buffer (Kurabo, Osaka, Japan). The mixture was centrifuged at $8,000 \mathrm{~g}$ for 10 minutes. The supernatant from each sample was then isolated and stored at $-80^{\circ} \mathrm{C}$ until analysis. We performed western blotting as previously described (19). Briefly, the supernatant was determined by protein assay. Equal amounts of protein were applied to $12 \%$ sodium dodecyl sulfate-polyacrylamide gel and separated by electrophoresis. The membranes were incubated at room temperature for 1 hour with primary antibodies against Clock (1:500; Santa Cruz Biotechnology, Santa Cruz, CA, USA), Bmal1 (1:1,000; Abcam), reverse 
orientation c-erb A gene (Rev-Erb)a (1:1,000; Medical \& Biological Laboratories; Nagoya, Aichi, Japan), nuclear factor, interleukin 3 regulated (NFIL-3) (1:1,000; Cell Signaling Technology, Danvers, MA, USA), the Th17 cell marker RAR-related orphan receptor gamma (RORYt; 1:100; Biorbyt, Cambridge, UK) and interleukin (IL)-17 (1:1,000; Aviva Systems Biology, San Diego, CA, USA) or $\beta$-actin $(1: 5,000$; Sigma-Aldrich, St. Louis, MO, USA). The membranes were washed and incubated with a horseradish peroxidase-conjugated secondary antibody (Novex, Frederick, MD, USA). The immune complexes were detected using ImmunoStar Zeta regent (Wako, Osaka, Japan), and the images were acquired using the Multi-Gauge software program (Fujifilm, Greenwood, SC, USA).

\section{Quantification of TSLP, TNF- $\alpha$ and IL-6 levels in plasma using an enzyme-linked immunosorbent assay (ELISA)}

Blood samples were taken on the fifth day after the start of DSS treatment. The plasma levels of TSLP, tumor necrosis factor (TNF)- $\alpha$, and IL-6 were determined using commercial enzyme-linked immunosorbent assay (ELISA) kits (TSLP and TNF-a: R\&D Systems, Minneapolis, MN, USA; IL-6: BioLegend, San Diego, CA, USA) in accordance with the manufacturers' instructions. The optical densities of the wells were measured with a microplate reader (Molecular Devices, Sunnyvale, CA, USA).

\section{Statistical analysis}

All data are presented as the mean \pm standard deviation (SD). Results were statistically analyzed using Microsoft Excel 2010, and with one-way analysis of variance (ANOVA) followed by Tukey's post hoc test using SPSS, version 20 (IBM, Armonk, NY, USA). The differences were considered statistically significant at $\mathrm{p}<0.05$.

\section{Results}

\section{Effect of UVB eye irradiation on DSS-treated mice}

Diarrhea and fecal bleeding were observed after DSS treatment in the UVB/DSS-treated mice. The disease activity scores of the UVB/DSS-treated mice were higher than those of the DSS-treated mice (Fig. 1A and 1D). DSS treatment also resulted in decreases in body weight and colon length. The average colon length of the UVB/DSS-treated mice was shorter than that of the DSS-treated mice (Fig. 1B and 1C).
Effect of UVB eye irradiation on the plasma levels of TSLP, IL-6, and TNF- $\alpha$ in DSS-treated mice

We performed ELISAs to measure the plasma levels of TSLP, IL-6, and TNF-a in the DSS-treated mice after 5 days of treatment. The plasma levels of TSLP, IL-6, and TNF-a were increased by DSS treatment. In the UVB/DSS-treated mice, the levels of TSLP and IL-6 were higher than in the DSS-treated mice (Fig. 2).

\section{Effects of UVB eye irradiation on the expression of Clock, Bmall, Rev-Erba, NFIL3, RORYt, and IL- 17 in DSS-treated mice}

Next, we measured the expression of the clock genes, IL-17, and the Th17 transcription factor RORyt, in order to investigate the cause of the increase in TSLP. In the UVB/DSS-treated mice, the expression of the clock genes Clock, Bmal1, and Rev-Erba was higher than in DSS-treated mice (Fig. 3). On the contrary, in the UVB/DSS-treated mice, the expression of NFIL3 was lower than in the DSStreated mice. The expression of ROR $\gamma t$ and IL-17 was higher in the UVB/DSS-treated mice than in the DSS-treated mice.

\section{Effect of UVB eye irradiation on the DSS-treated mice after PF670462 administration}

The PF670462/UVB/DSS-treated mice had lower disease activity scores than the UVB/ DSS-treated mice (Fig. 4). However, PF670462 did not lower the disease activity scores of UVB/DSS-treated mice to those of the DSS-treated mice.

\section{Effects of UVB eye irradiation on the expression of Bmal1, Clock, Rev-Erba, NFIL3, IL-17, and RORyt in the DSS-treated mice after PF670462 administration}

The expression of Bmal1, Clock and Rev-Erba in the PF670462/UVB/DSS-treated mice was lower than in the UVB/DSS-treated mice (Fig. 5). The expression of NFIL3 in the PF670462/UVB/DSS-treated mice was higher than in the UVB/DSS-treated mice, and similar to that in the DSS-treated mice. Furthermore, the expression of RORyt and IL-17 in the PF670462/UVB/DSS-treated mice was lower than in the UVB/DSS-treated mice, but not reduced to the levels in the DSS-treated mice. 
A
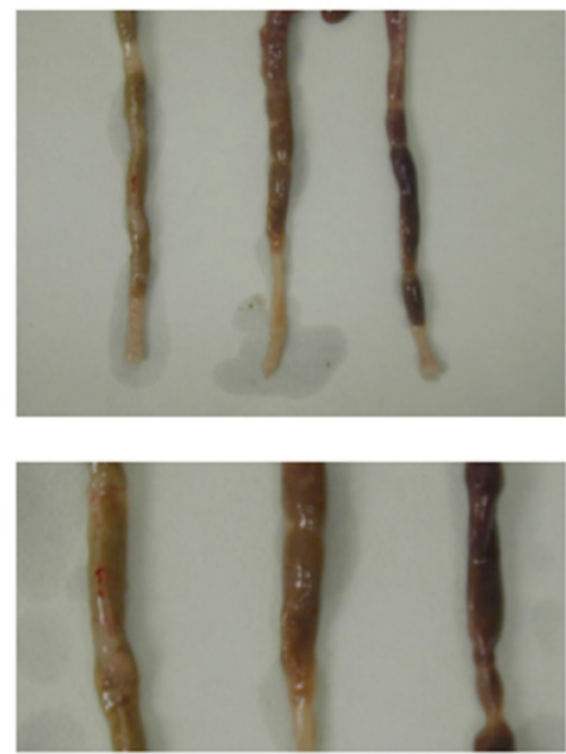

Control DSS UVB+DSS

C

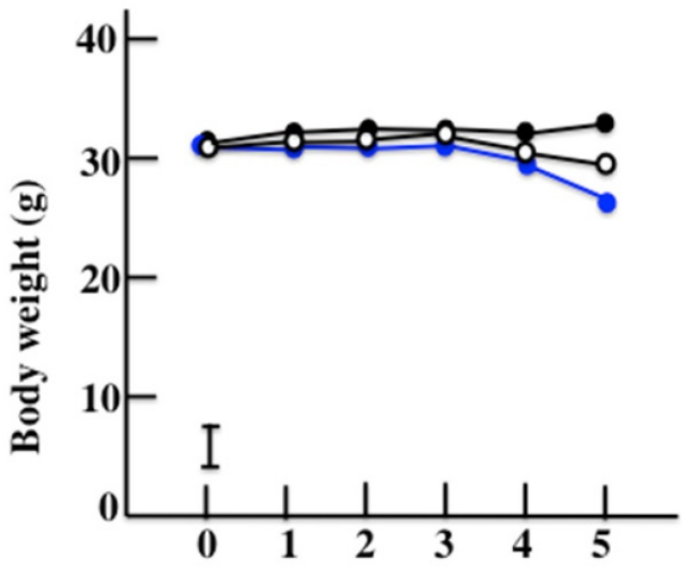

B

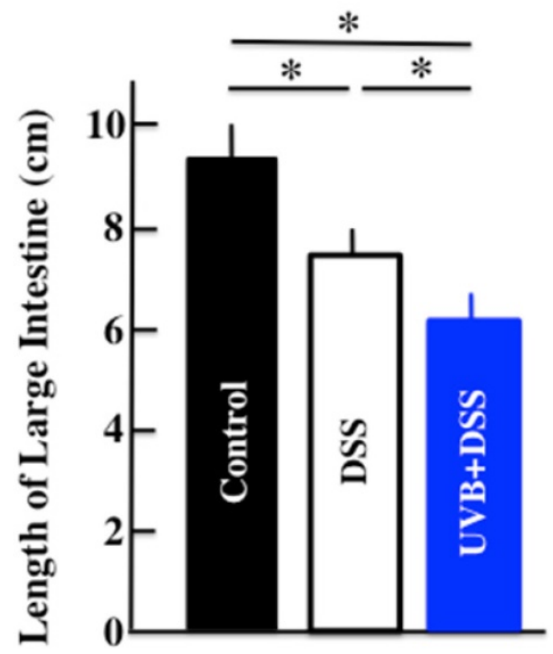

5 days after DSS treatment

D

- Control

- DSS

- DSS+UVB

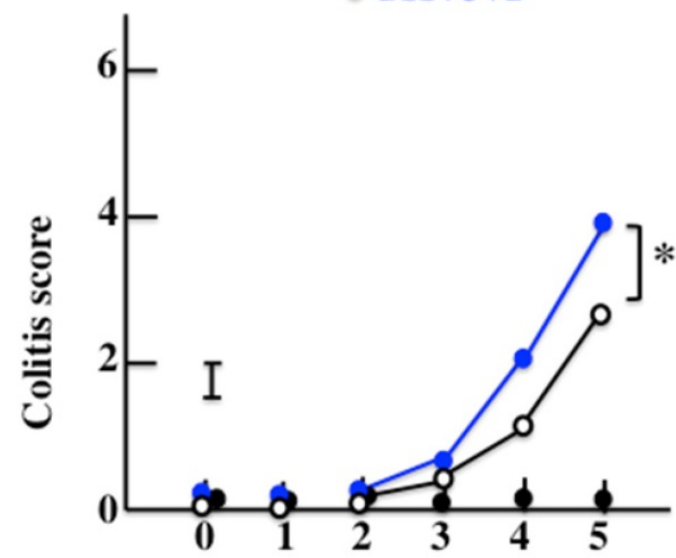

Day after DSS treatment

Figure 1. The effects of UVB eye irradiation on DSS-induced ulcerative colitis. The length of the large intestine (A, B). The body weights (C) and colitis scores (D) over the course of the experiment. The values represent the mean \pm SD derived from 6 animals $(B)$ or the mean and pooled standard error of the mean (C, D). ${ }^{*} \mathrm{p}<0.05$ (DSS vs. UVB/DSS).
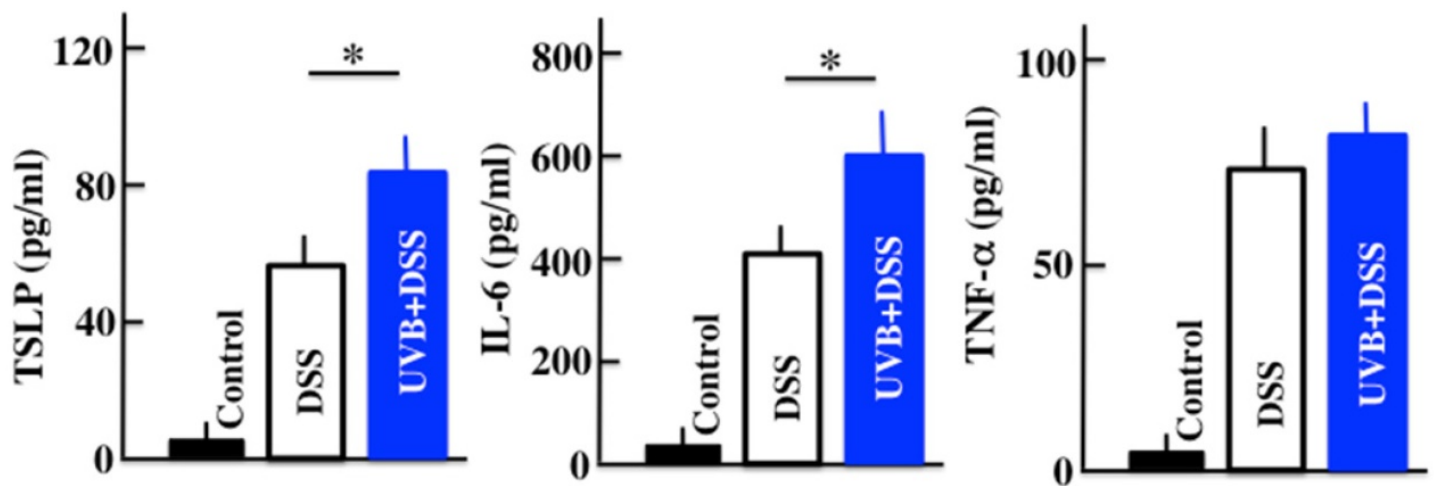

Figure 2. Analysis of the plasma TSLP, IL-6, and TNF- $\alpha$ concentrations after UVB/DSS-treatment. The values represent the mean \pm SD derived from 6 animals. $*_{\mathrm{p}}<0.05$ (DSS vs. UVB/DSS). 

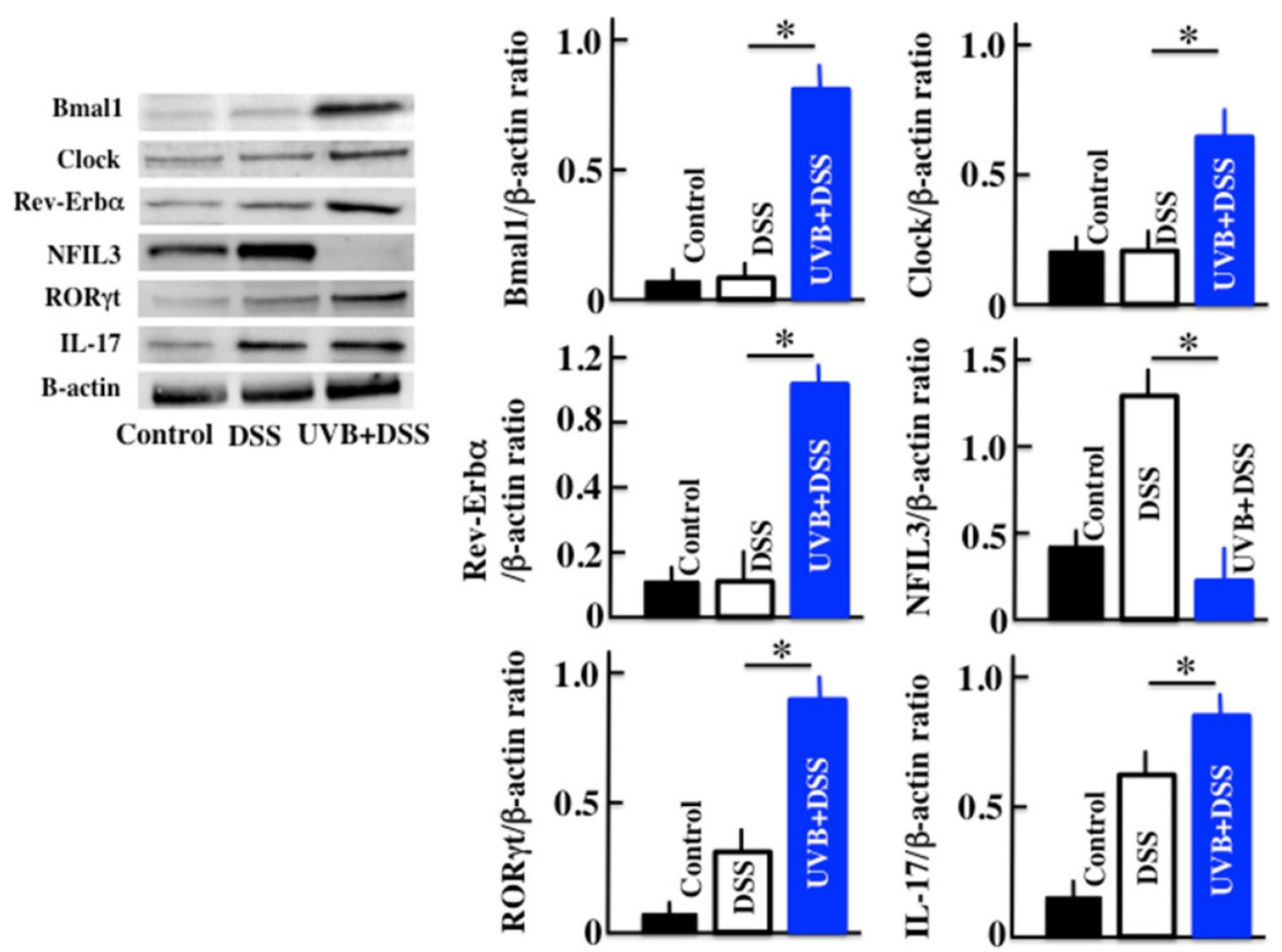

5 days after DSS treatment

Figure 3. Western blot analysis of Bmall, Clock, Rev-Erba, NFIL3, RORyt, and IL-17 in the colon. The values represent the mean \pm SD derived from 6 animals. $*_{\mathrm{p}}<0.05$ (DSS vs. UVB/DSS).
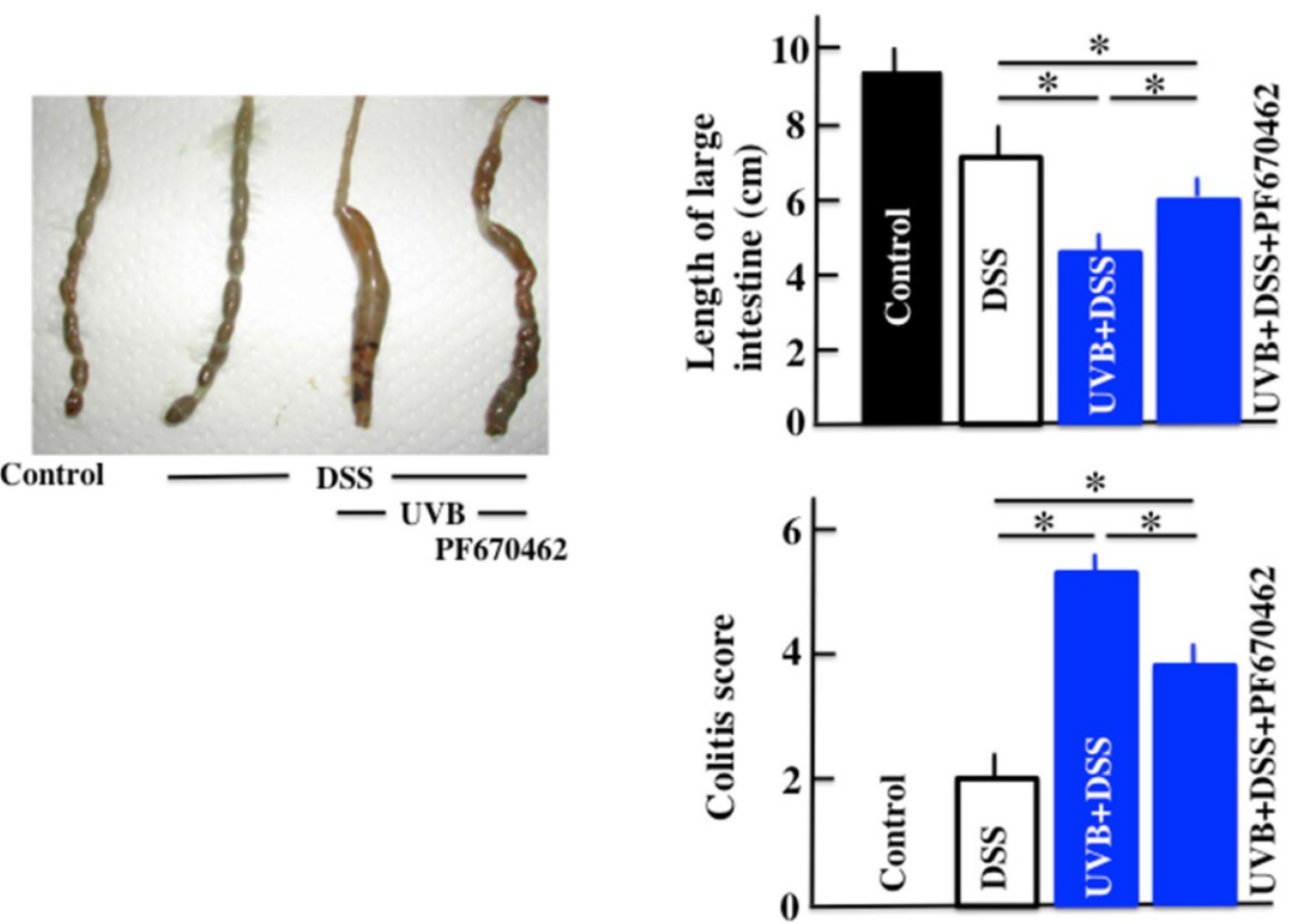

5 days after DSS treatment

Figure 4. The effects of PF670462 on the colitis score and length of the large intestine in UVB/DSS-treated mice. PF670462 is PER2 activator. The values represent the mean \pm SD. ${ }^{*} p<0.05$. 

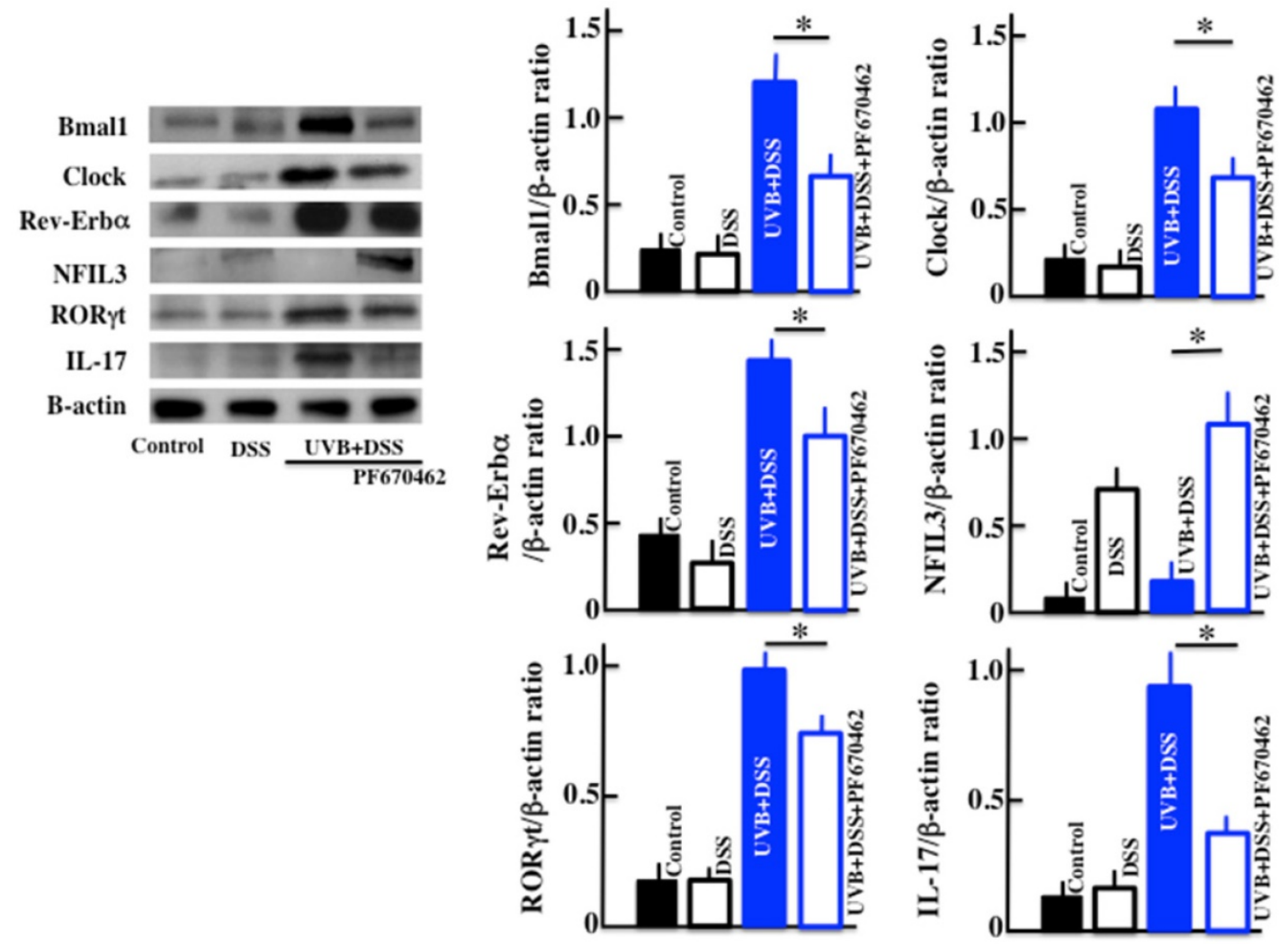

5 days after DSS treatment

Figure 5. The effects of PF670462 on the expression of Bmal I, Clock, Rev-Erba, NFIL3, RORYt, and IL-17 in the colons of the UVB/DSS-treated mice. The values represent the mean \pm SD derived from 6 animals. ${ }^{*} p<0.05$ (DSS vs. UVB/DSS).

\section{Discussion}

In this study, UVB eye irradiation exacerbated DSS-induced ulcerative colitis in mice by inducing increases in the levels of TSLP, IL-6, and TNF-a in plasma, and IL-17 in the colon. UVB eye irradiation increased the expression of the clock genes Clock, Bmal1, and Rev-Erba, in the colon, but decreased the expression of NFIL3. An antagonist of Clock/Bmal1 inhibited the UVB eye irradiation-induced deterioration of the colitis.

We reported in a previous study that UVB eye irradiation exacerbated DSS-induced ulcerative colitis (10). ACTH, secreted from the hypothalamus and pituitary gland, was increased by UVB eye irradiation, as was the expression of melanocortin 2 receptor (MC2R) in the colon. ACTH signaled through the receptor to induce the secretion of corticosterone, and induced the secretion of inflammatory cytokines, such as IL-18, IL-6, and histamine. However, treatment with RU486, a glucocorticoid receptor antagonist, only partially inhibited the UVB eye irradiation-mediated deterioration of DSS-induced ulcerative colitis. These findings indicated that additional mechanisms mediated the effects of UVB eye irradiation in
DSS-induced ulcerative colitis.

Light regulates the circadian rhythms of animals, thereby regulating allergy. In the examination of a circadian rhythm, the time of a sampling becomes important. In this study, we obtained colon samples at morning (ZT2-4), because there is a report that the levels of Bmal, Clock and Rev-Erba $\alpha$ were remarkably high at morning (20). In addition, since we examined in this time on our previous study, we also done at the same time (10). NFIL3 controls the function of inflammatory immune cells, and the expression of NFIL3 inhibits inflammatory bowel disease $(21,22)$. Furthermore, the intestines of NFIL3-deficient mice are characterized by increased numbers of Th17 cells compared to those in wild type mice, as well as the development of the symptoms of inflammatory bowel disease (23). In this study, the increased expression of Clock/Bmal1 and Rev-Erba in DSS-treated mice upon UVB eye irradiation decreased the expression of NFIL3, which increased the expression of ROR $\gamma t$, the Th17 transcription factor. Therefore, we hypothesized that UVB eye irradiation increased Th17 cell differentiation (23). In this study, we observed similar alterations in the expression of the clock genes and RORyt. Furthermore, the amelioration of the effects of UVB eye irradiation by the inhibition of Clock/Bmal1 


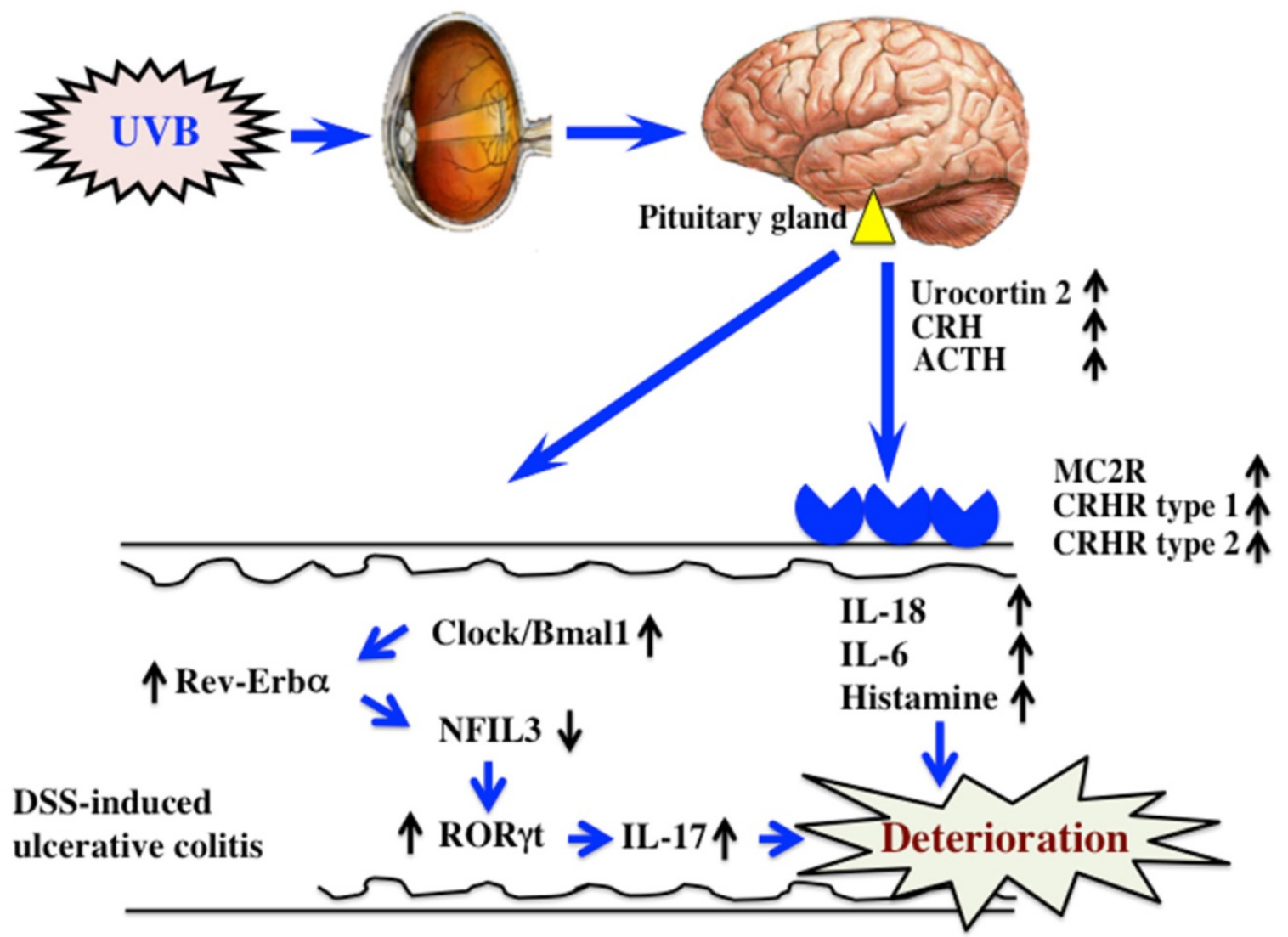

Figure 6. The mechanism of UVB eye irradiation-mediated exacerbation of DSS-induced ulcerative colitis.

(CK1 $\varepsilon / \delta$ inhibitor: PF670462) was accompanied by increased expression of PER2. The expression of the clock gene did not change with single administrations of $\mathrm{CK} 1 \varepsilon / \delta$ inhibitor (data not shown). However, the period of the circadian rhythm is extended by the administration of $\mathrm{CK} 1 \varepsilon / \delta$ inhibitor (14). Because in this study we do not see only one point of the period, but detailed investing is required. Thus, variations in the clock genes caused by UVB eye irradiation appear to influence the symptoms of DSS-induced colitis. However, we do not yet understand the detailed relationship between UVB eye irradiation and the clock genes. In addition, since the clock genes are regulated by circadian rhythms, the timing of the radiation is likely to be important. Therefore, further study is necessary to determine the relationship between UVB eye irradiation and clock genes, with particular consideration given to the time course of UVB irradiation (morning, afternoon and evening).

\section{Conclusion}

DSS-induced ulcerative colitis appears to be exacerbated by UVB eye irradiation via regulation of a hormone of pituitary gland origin (10) and clock genes (Fig. 6). Clock genes are not only affected by UVB eye irradiation, but also by disturbances in lifestyle and physical stress. Therefore, the factors that alter the expression of clock genes are also likely to directly affect ulcerative colitis. In modern society, the alteration of clock genes is frequently observed in perturbed states. Therefore, correcting disturbances to the clock genes may form the basis of treatments for various diseases.

\section{Acknowledgements}

This work was supported by JSPS KAKENHI Grant Number 18K11085.

\section{Competing Interests}

The authors have declared that no competing interest exists.

\section{References}

1. Hiramoto $\mathrm{K}$, Tanaka H, Yanagihara N, Sato EF, Inoue M. Effect of 17beta-estradiol on immunosuppression induced by ultraviolet B irradiation. Arch Dermatol Res. 2004; 295: 307-311.

2. Reeve VE, Bosnic M, Boehm-Wilcox C, Nishimura N, Ley RD. Ultraviolet A radiation (320-400 $\mathrm{nm}$ ) protects hairless mice from immunosuppression induced by ultraviolet B radiation $(280-320 \mathrm{~nm})$ or cis-urocanic acid. Int Arch Allergy Immunol. 1998; 115: 316-322.

3. Makinen M, Stenback F. Skin tumor development and keratin expression in different experimental models. Relation to inducing agent and target tissue structure. Exp Toxicol Pathol. 1998; 50: 199-208.

4. Yagi H, Tokura Y, Wakita H, Furukawa F, Takigawa M. TCRV beta7 + Th2 cells mediate UVB-induced suppression of murine contact photosensitivity by releasing IL-10. J Immunol. 1996; 156: 1824-1831.

5. Mizuno K, Okamoto H, Horio T. Ultraviolet B radiation suppresses endocytosis, subsequent maturation, and migration activity of langerhans cell-like dendritic cells. J Invest Dermatol. 2004; 122: 300-306. 
6. Simon JC, Cruz Jr PD, Bergstresser PR, Tigelaar RE. Low dose ultraviolet B-irradiated Langerhans cells preferentially activate CD4+ cells of the T helper 2 subset. J Immunol. 1990; 145: 2087-2091.

7. Nishigori C, Yarosh DB, Ullrich SE, Vink AA, Bucana CD, Roza L, Kripke ML. Evidence that DNA damage triggers interleukin 10 cytokine production in UV-irradiated murine keratinocytes. Proc Natl Acad Sci USA. 1996; 93: 10354-10359.

8. Mutou Y, Ibuki Y, Kojima S. Immunomodulatory effects of ultraviolet B irradiation on atopic dermatitis in NC/Nga mice. Photodermatol Photoimmunol \& Photomed. 2007; 23: 135-144.

9. Meduri NB, Vandergriff T, Rasmussen H, Jacobe H. Phototherapy in the management of atopic dermatitis: a systematic review. Photodermatol Photoimmunol Photomed. 2007; 23: 106-112.

10. Hiramoto $\mathrm{K}$, Yamate $\mathrm{Y}$, Sato EF. The effects of ultraviolet eye irradiation on dextran sodium sulfate-induced ulcerative colitis in mice. Photochem Photobiol. 2016; 92: 728-734.

11. Nakamura Y, Nakano N, Ishimaru K, Ando N, Katoh R, Suzuki-Inoue K, et al. Inhibition of IgE-mediated allergic reactions by pharmacologically targeting the circadian clock. J Allergy Clin Immunol. 2016; 137:1226-1235.

12. Numano R, Yamazaki S, Umeda N, Samura T, Sujino M, Takahashi R, et al. Constitutive expression of the Period1 gene impairs behavioral and molecular circadian rhythms. Proc Natl Acad Sci USA. 2006; 103: 3716-3721.

13. Yamazaki S, Numano R, Abe M, Hida A, Takahashi R, Ueda M, et al. Resetting central and peripheral circadian oscillators in transgenic rats. Science. 2000; 288: 682-685.

14. Badura L, Swanson T, Adamowicz W, Adams J, Cianfrogna J, Fisher J, et al. An inhibitor of casein kinase I epsilon induces phase delays in circadian rhythms under free-running and entrained conditions. J Pharmacol Exp Ther. 2007; 322: 730-738.

15. Geyfman M, Kumar V, Liu Q, Ruiz R,Gorden W, Espitia F, et al. Brain and muscle Arnt-like protein-1 (BMAL1) controls circadian cell proliferation and susceptibility to UVB-induced DNA damage in the epidermis. Proc Natl Acad Sci USA. 2012; 109: 11758-11763.

16. Yamate Y, Hiramoto K, Kasahara E, Sato EF. UVA irradiation of the eye modulates the contact hypersensitivity of the skin and intestine by affecting mast cells in mice. Photodermatol Photoimmunol Photomed. 2015; 31: 129-140.

17. Hiramoto K, Yamate Y, Sugiyama D, Takahashi Y, Mafune E. Tranexamic acid suppresses ultraviolet B eye irradiation-induced melanocyte activation by decreasing the levels of prohormone convertase 2 and alpha-melanocyte-stimulating hormone. Photodermatol Photoimmunol Photomed. 2014; 30: 302-307.

18. Nishimura T, Andoh A, Hashimoto T, Kobori A, Tsujikawa T, Fujiyama Y. Cellobiose prevents the development of dextran sulfate sodium (DSS)-induced experimental colitis. J Clin Biochem Nutr. 2010; 46: 105-110.

19. Hiramoto K, Sugiyama D, Takahashi Y, Mafune E. The amelioration effect of tranexamic acid in wrinkles induced by skin dryness. Biomed Pharmacother. 2016; 80: 16-22.

20. Shimba S. Reulation of skin functions by the moleculer clock system. Ann Report Cosmetol. 2016; 24: 117-123.

21. Kobayashi T, Matsuoka K, Sheikh SZ, Elloumi HZ, Kamada N, Hisamatsu T, et al. NFIL3 is a regulator of IL-12 p40 in macrophages and mucosal immunity. J Immunol. 2011; 186: 4649-4655.

22. Kobayashi T, Steinbach EC, Russo SM, Matsuoka K, Nochi T, Maharshak N, et al. NFIL3-deficient mice develop microbiota-dependent, IL-12/23-driven spontaneous colitis. J Immunol. 2014; 192: 1918-1927.

23. Yu X, Rollins D, Ruhn KA, Stubblefield JJ, Green CB, Kashiwada M, et al. TH17 cell differentiation is regulated by the circadian clock. Science. 2013; 342: $727-730$. 